\title{
Coexistent Membranous Nephropathy with Doubly ANCA-Associated Crescentic Glomerulonephritis: A Case Report and Review of Literature
} Sirisak Chanprasert ${ }^{1}$, Wisit Cheungpasitporn ${ }^{1 *}$ and Ann K Eldred ${ }^{2}$

${ }^{1}$ Department of Internal Medicine, Bassett Medical Center and Columbia University College of Physicians and Surgeons, Cooperstown, New York 13326, USA ${ }^{2}$ Department of Nephrology and Dialysis, Bassett Medical Center and Columbia University College of Physicians and Surgeons, Cooperstown, New York 13326, USA

\begin{abstract}
Introduction: Membranous nephropathy $(\mathrm{MN})$ is the most common causes of the nephrotic syndrome in nondiabetic, Caucasian adults. Pauci-immune necrotizing and crescentic glomerulonephritis (PNCGN) typically present with rapidly progressive glomerulonephritis. Coexistent MN and PNCGN is a rare occurrence. We report a case of both MPO- and PR3-ANCA associated NCGN with MN that presented as rapidly progressive glomerulonephritis.
\end{abstract}

Case presentation: A 46-year-old female presented with nausea and vomiting. On physical examination, the patient was a febrile and normotensive. Blood tests showed acute kidney injury and anemia. Urinalysis demonstrated numerous dysmorphic red blood cells with granular casts and nephrotic range proteinuria. Further testing showed negative ANA, positive anti-dsDNA, PR3-ANCA and MPO-ANCA. Kidney biopsy revealed the diagnosis of concurrent PNCGN with membranous nephropathy. The diagnosis of concurrent ANCA-associated NCGN with Membranous nephropathy was made. High dose intravenous methyl prednisolone was initiated. Unfortunately, the patient developed diffuse alveolar hemorrhage and underwent 6 cycles of plasmapheresis, intravenous Cyclophosphamide and pulse dose steroids with transitioned to oral prednisone and mycophenolate. On follow up, her disease seemed to be well suppressed without dialysis.

Conclusion: Membranous nephropathy with PNCGN is a rare concurrent glomerulopathy, and even more rare with both MPO and PR-3 positivity. The diagnosis of MN with PNCGN should be considered in patients who present with RPGN and nephrotic range proteinuria.

Keywords: Acute kidney injury; ANCA-associated crescentic glomerulonephritis; Membranous nephropathy

Abbreviation: ANA: Anti Nuclear Antibody; ANCA: Antineutrophil cytoplasmic antibody; Anti-dsDNA: Anti-Double Stranded DNA Antibody; C3: Complement 3; C4: Complement 4; CT: computed tomography; ESRD: End Stage Renal Disease; GN: Glomerulonephritis; MN: Membranous Nephropathy; MPO: Myeloperoxidase; MTP: metatarsophalangeal; PNCGN: Pauci-immune crescentic glomerulonephritis; PR-3: Proteinase-3; RPGN: Rapidly progressive glomerulonephritis.

\section{Introduction}

Membranous nephropathy ( $\mathrm{MN}$ ) is the most common cause of primary nephrotic syndrome in nondiabetic, Caucasian adults, accounting for more than one third of cases [1]. Most of patients with $\mathrm{MN}$ have preserved renal function at the time of presentation. Renal failure usually develops gradually in patients with $\mathrm{MN}$ and only rarely is complicated by acute kidney injury. Pauci-immune necrotizing and crescentic glomerulonephritis (PNCGN) typically present with rapidly progressive glomerulonephritis (RPGN). Coexistent MN and PNCGN is a rare occurrence. We report a case of both MPO- and PR3-ANCA associated NCGN with membranous nephropathy that presented as rapidly progressive glomerulonephritis.

\section{Case Presentation}

A 46-year-old Caucasian woman presented to emergency department with nausea, vomiting and weight loss. Medical history was remarkable for gastroesophageal reflux disease (GERD), fibromyalgia and depression. She noticed poor appetite and 40-pound weight loss over 2 months. Patient denied any recent history of upper respiratory tract infection, or skin infection. She was taking 4,800 $\mathrm{mg}$ of ibuprofen per day and no other medications. On physical examination, the patient was a febrile, normotensive with a blood pressure of $120 / 80$ $\mathrm{mmHg}$ and her urine output over $24 \mathrm{~h}$ was approximately $2 \mathrm{~L}$ of dark red urine. There were multiple tiny ischemic lesions on the distal part of fingers, more pronounced on both thumbs. There was no active synovitis appreciated.

Laboratory testing showed normocytic anemia (HGB $8.6 \mathrm{gm} /$ dL, HCT 25.1\%, MCV 89.7fL, and MCH $28.5 \mathrm{pg}$ ), elevated serum creatinine of $4.1 \mathrm{mg} / \mathrm{dl}$ (base line $0.8 \mathrm{mg} / \mathrm{dL}$ ), serum albumin level of $3.0 \mathrm{~g} / \mathrm{dL}$, total cholesterol $95 \mathrm{mg} / \mathrm{dL}$, and triglycerides $147 \mathrm{mg} /$ dL. Microscopic urinalysis demonstrated numerous dysmorphic red blood cells, granular casts. She had nephrotic range proteinuria with a urine protein to creatinine ratio of 3.68 and UPEP only notable for albuminuria. Renal ultrasound disclosed no hydronephrosis with normal sized kidney $10.7 \mathrm{~cm}$ on the right and $10.3 \mathrm{~cm}$ on the left. The possibility of rapidly progressive glomerulonephritis from systemic vasculitis was raised by the nephrology evaluation. Interestingly, further testing showed a negative ANA, highly positive anti-dsDNA of $>45.0 \mathrm{IU} / \mathrm{ml}$, positive PR3- and MPO-ANCA (both $>8.0$; Mayo Clinic laboratory), and low level of C3 and C4 (60 and $11.5 \mathrm{mg} / \mathrm{dl}$, respectively). Other laboratory tests including $\mathrm{HBsAg}$, anti-HCV, anti-HIV, rheumatoid Factor, anti-CCP antibody and anti-GBM

*Corresponding author: Wisit Cheungpasitporn, Department of Internal Medicine Bassett Medical Center and Columbia University College of Physicians and Surgeons, Cooperstown, New York 13326, USA, E-mail: wisit.cheungpasitporn@ bassett.org

Received October 24, 2011; Accepted November 15, 2011; Published November 17,2011

Citation: Chanprasert S, Cheungpasitporn W, Eldred AK (2011) Coexistent Membranous Nephropathy with Doubly ANCA-Associated Crescentic Glomerulonephritis: A Case Report and Review of Literature. J Nephrol Therapeutic 1:106. doi:10.4172/2161-0959.1000106

Copyright: () 2011 Chanprasert S, et al. This is an open-access article distributed under the terms of the Creative Commons Attribution License, which permits unrestricted use, distribution, and reproduction in any medium, provided the original author and source are credited. 
were negative. Serum protein electrophoresis showed polyclonal gammopathy and low albumin. During the course of admission the patient developed palpable purpura. Dermatology consultation was requested. The pathological findings of skin biopsy were consistent with leukocytoclastic vasculitis. PNCGN with glomerulonephritis was the most likely diagnosis at that point. However, since the anti-dsDNA was highly positive and the $\mathrm{C} 3$ and $\mathrm{C} 4$ levels were low, systemic lupus erythematosus (SLE) was also in the differential diagnosis.

A renal biopsy with CT-guided was then performed (Figure 1), and sampling for light microscopy included 29 glomeruli, four of which were globally sclerotic. The results showed focal segmental necrotizing and crescentic glomerulonephritis. Immunofluorescence revealed granular global capillary wall positivity of 1-2+ intensity for IgG, C3, kappa, and lambda. Electron microscopy revealed minute, stage 1, predominantly segmental subepithelial deposits as well as infrequent segmental mesangial deposits. These findings were consistent with focal segmental necrotizing and crescentic glomerulonephritis, pauciimmune type with membranous glomerulopathy, segmental, stage 1 .

The diagnosis of concurrent PNCGN (both PR3- and MPOANCA) with membranous nephropathy was made. High dose intravenous methylprednisolone was initiated for 3 days, and cyclophosphamide was ordered after the biopsy result was observed. Unfortunately, the patient developed diffuse alveolar hemorrhage. The patient was incubated and then transferred to an outside hospital,

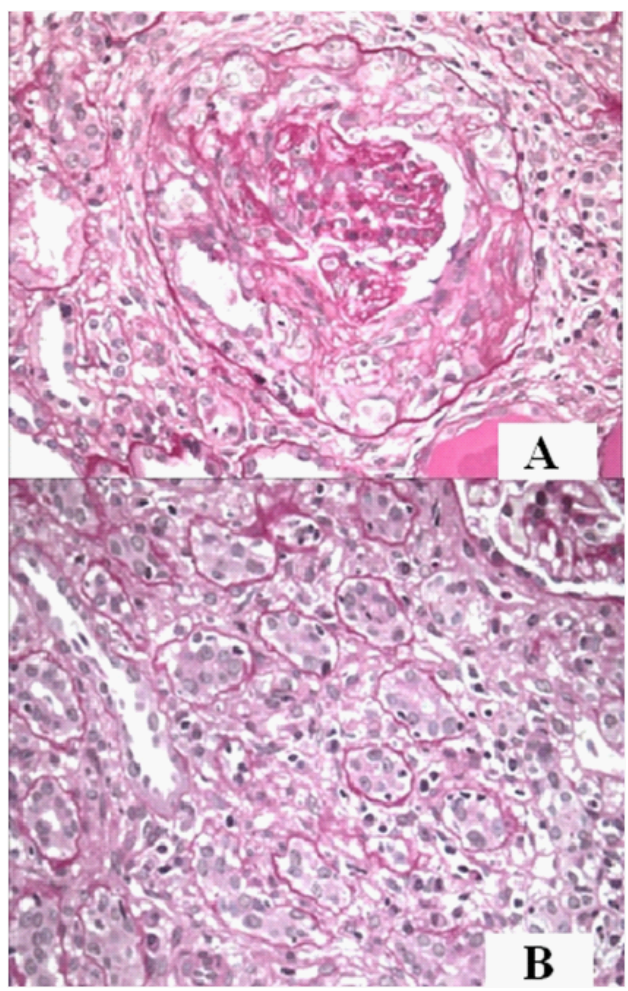

Figure 1: (A) Sampling for light microscopy reveals a focal segmental necrotizing \& crescentic glomerulonephritis, typical of what may be seen in the setting of ANCA seropositivity. (B) The findings are also notable for moderate to severe interstitial inflammation and extensive tubulitis which also likely relate to ANCA seropositivity. Immunofluorescence reveals granular global capillary wall positivity of 1-2+ intensity for IgG, C3, kappa, and lambda. Electron microscopy reveals minute, stage 1 , predominantly segmental subepithelial deposits as well as infrequent segmental mesential deposits. Taken together, these findings appear sufficient to establish the Coexistent MN and ANCA-associated NCGN. in order to receive plasmapheresis. She received hemodialysis and underwent 6 cycles of plasmapheresis, intravenous cyclophosphamide (CY) and pulse dose steroids. She was transitioned to oral prednisone and mycophenolate mofetil. After the hospital discharge, the patient was feeling well. She was asymptomatic. She denied leg swelling, shortness of breath, hemoptysis, cough or sputum production. On recent follow up, 6 months after diagnosis, she continues to do well. Serum creatinine decreased to $1.9 \mathrm{mg} / \mathrm{dL}$ while proteinuria decreased to $2.1 \mathrm{~g} / 24 \mathrm{~h}$. Both PR3- and MPO-ANCA level decreased to 4.4 and 4.6 respectively (Mayo Clinic laboratory).

\section{Discussion}

Membranous nephropathy is characterized histologically by the formation of subepithelial immune complex deposits with resultant changes to the glomerular basement membrane (GBM), most notably GBM spike formation [2]. Fibrinoid necrosis and crescent formation is rarely seen in membranous nephropathy, except in those cases associated with systemic lupus erythematosus, corresponding to ISN/ RPS lupus nephritis class III and V or IV and V [3,4], hepatitis B or $C$ virus infection and treatment with penicillamine, hydralazine and propylthiouracil [5-8]. In general, the absence of evidence of SLE, findings of $\mathrm{MN}$ with necrosis and crescent formation should raise the possibility of two potential superimposed disease processes, anti-GBM disease and ANCA-associated NCGN [9]. In our case vignette, SLE was also in the differential. Ten percents of SLE patients may have negative ANA and positive anti-dsDNA. The dominant process on biopsy as well as the clinical finding; including pulmonary hemorrhage, support PNCGN as the prominent process. We cannot be sure she does not also have lupus, ISN/RPS 2004 class V.

Unlike MN, which often has an insidious course progressing to renal failure over many years, patients with superimposed crescentic GN generally have a more aggressive clinical course and may present with or progress rapidly to renal failure $[10,11]$. These patients may present with a rapidly progressive glomerulonephritis or develop a nephritic picture after initially presenting with a nephrotic syndrome.

Coexistent MN and PNCGN is a rare occurrence, with only 25 reported cases in the English literature in which clinical and pathologic findings are detailed [9,12-18]. Thirteen patients had P-ANCA by indirect immune fluorescent (IIF) staining, seven of whom were tested with ELISA and found to have MPO-ANCA. Eight patients had C-ANCA by IIF. Two patients were tested with ELISA only and were found to have MPO-ANCA. The remaining two patients, one had both MPO- and PR3- ANCA and the other had an atypical ANCA. Our case presentation was the second case report of both MPO- and PR3ANCA-associated NCGN with membranous nephropathy.

Most of patients with MN and PNCGN were diagnosed simultaneously at presentation as was our case vignette. On the other hand, the situation of concurrent MN and anti-GBM disease, in which MN preceded the development of anti-GBM nephritis in close to $50 \%$ of reported cases $[19,20]$. The reason for concurrent MN and PNCGN is unclear. A report of all biopsies received between 2000 and 2008 at a high-volume renal pathology unit found 14 cases in which both MN and PNCGN were detected. Based on the expected incidences for each disease entity in this population, the authors concluded that the co-existence of MN and PNCGN was coincidental [9]. However, Hanamura et al. [21] recently reported that myeloperoxidase may form immune complexes and develop membranous nephropathy-like lesions in some cases of PNCGN [21]. 
Citation: Chanprasert S, Cheungpasitporn W, Eldred AK (2011) Coexistent Membranous Nephropathy with Doubly ANCA-Associated Crescentic Glomerulonephritis: A Case Report and Review of Literature. J Nephrol Therapeutic 1:106. doi:10.4172/2161-0959.1000106

Page 3 of 5

Patients with both MN and PNCGN are likely to have heavier proteinuria than patients with PNCGN alone. All of 25 reported cases with urine output had proteinuria [9,12-18]. From the recent report, the mean 24-h urine protein for these patients was $6.5 \mathrm{~g}$, compared with 1.7 to $2.5 \mathrm{~g}$ in patients with ANCA disease alone [22]. Hematuria was documented in all but one patient, who was oliguric. In our case presentation, the patient also had hematuria and nephrotic range proteinuria with a urine protein to creatinine ratio of 3.68 .

Although the treatment of MN is often supportive, renal vasculitis characteristically responds well to treatment with cyclophosphamide (CY) and prednisone [11]. Among the 25 reported cases of $\mathrm{MN}$ and PNCGN, induction therapy consisted of prednisone and CY in 20 patients, prednisone and azathioprine in one patient, and prednisone alone in one patient. One patient received mycophenolate mofetil and two patients also received plasmapheresis $[9,12-18]$.

Patients with MN and PNCGN have outcomes that appear to be worse than that of other patients with $\mathrm{MN}$ alone, with $50 \%$ reaching endpoints of death or ESRD [9]. Most of the reported cases needed dialysis and in one case the lesion of membranous nephropathy with crescents recurred after renal transplantation.

As a conclusion, although the association of PNCGN and MN is rare, it has a more aggressive clinical course when compared with MN alone. However, it is unclear if this dual glomerulopathy does worse than PNCGN alone. The diagnosis of MN with PNCGN should be considered in patients who present with RPGN and nephrotic range proteinuria.

\section{Consent}

The patient provided written consent for the publication of this case report and any accompanying images. A copy of the written consent is available for review by the journal's Editor-in-Chief.

\section{Acknowledgement}

We acknowledge Dr. Glen S. Markowitz, Professor of Clinical Pathology and Cell Biology, who analyzed the kidney biopsy and provided us the biopsy imaging and Dr.Edward Bischof, Program Director of the Internal Medicine Residency Program at Bassett Medical Center, who always encourages us to study our patients cases.

\section{References}

1. Korbet SM, Genchi RM, Borok RZ, Schwartz MM (1996) The racial prevalence of glomerular lesions in nephrotic adults. Am J Kidney Dis 27: 6 47-651.

2. Glassock RJ, Adler SG, Ward HJ, Cohen AH, Brenner BM, Rector FC (1986) Primary glomerular disease. The Kidney, Saunders, Philadelphia, 929-1013.

3. Pasquali S, Banfi G, Zucchelli A, Moroni G, Ponticelli C, et al. (1993) Lupus membranous nephropathy: long term outcome. Clin Nephrol 39: 175-182.

4. Schwarz MM, Heptinstall RH (1992) Membranous glomerulonephritis. Pathology of the Kidney, Little, Brown, Boston 559-562.

5. Bienaimé F, Clerbaux G, Plaisier E, Mougenot B, Ronco P, et al. (2007) D-Penicillamine-induced ANCA-associated crescentic glomerulonephritis in Wilson disease. Am J Kidney Dis 50: 821-825.

6. Vozmediano C, Sánchez de la Nieta MD, González L, Alvarez T, de la Torre $\mathrm{m}$, et al. (2005) Membranous nephropathy and crescentic glomerulonephritis. Nefrologia 25: 328-331

7. Stokes MB, Foster K, Markowitz GS, Ebrahimi F, Hines W, et al. (2005) Development of glomerulonephritis during anti-TNF-alpha therapy for rheumatoid arthritis. Nephrol Dial Transplant 20: 1400-1406.

8. Mathieson PW, Peat DS, Short A, Watts RA (1996) Coexistent membranous nephropathy and ANCA-positive crescentic glomerulonephritis in association with penicillamine. Nephrol Dial Transplant 11: 863-866.
9. Nasr SH, Said SM, Valeri AM, Stokes MB, Masani NN, et al. (2009) Membranous glomerulonephritis with ANCA-associated necrotizing and crescentic glomerulonephritis. Clin J Am Soc Nephrol. 4: 299-308.

10. Taniguchi Y, Yorioka N, Kumagai J, Ito T, Yamakido M, (1999) Myeloperoxidase antineutrophil cytoplasmic antibody-positive necrotizing crescentic glomerulonephritis and membranous glomerulonephropathy. Clin Nephrol. 52 253-255.

11. Tse WY, Howie AJ, Adu D, Savage CO, Richards NT, et al. (1997) Association of vasculitic glomerulonephritis with membranous nephropathy: a report of 10 cases. Nephrol Dial Transplant 12:1017-1027.

12. Gaber LW, Wall BM, Cooke CR (1993) Coexistence of anti-neutrophil cytoplasmic antibody-associated glomerulonephritis and membranous glomerulopathy. Am J Clin Pathol 99:211-215.

13. Taniguchi $\mathrm{Y}$, Yorioka N, Kumagai J, Ito T, Yamakido M, et al. (1999) Myeloperoxidase antineutrophil cytoplasmic antibody-positive necrotizing crescentic glomerulonephritis and membranous glomerulonephropathy. Clin Nephrol 52: 253-255.

14. Kanahara K, Yorioka N, Nakamura C, Kyuden Y, Ogata S, et al. (1997) Myeloperoxidase-antineutrophil cytoplasmic antibody-associated glomerulonephritis with membranous nephropathy in remission. Intern Med 36 841-846.

15. Dwyer KM, Agar JW, Hill PA, Murphy BF (2001) Membranous nephropathy and anti-neutrophil cytoplasmic antibody-associated glomerulonephritis: A report of 2 cases. Clin Nephrol 56: 394-397.

16. Tse WY, Howie AJ, Adu D, Savage CO, Richards NT, et al. (1997) Association of vasculitic glomerulonephritis with membranous nephropathy: A report of 10 cases. Nephrol Dial Transplant 12: 1017-1027.

17. Suwabe T, Ubara Y, Tagami T, Sawa N, Hoshino J, et al. (2005) Membranous glomerulopathy induced by myeloperoxidase-anti-neutrophil cytoplasmic antibody-related crescentic glomerulonephritis. Intern Med 44: 853-858.

18. Granata A, Floccari F (2011) Membranous glomerulonephritis with superimposed ANCA-associated vasculitis: another case report. NDT Plus 4: 80-81.

19. Nasr SH, llamathi ME, Markowitz GS, D’Agati VD (2003) A dual pattern of immunofluorescence positivity. Am J Kidney Dis 42: 19-26.

20. Nayak SG, Satish R (2007) Crescentic transformation in primary membranous glomerulopathy: association with anti-GBM antibody. Saudi J Kidney Dis Transpl 18: 599-602.

21. Hanamura K, Tojo A, Kinugasa S, Asaba K, Onozato ML, et al. (2011) Detection of myeloperoxidase in membranous nephropathylike deposits in patients with anti-neutrophil cytoplasmicantibodyassociated glomerulonephritis. Hum Pathol 42: 649-658.

22. Chen M, Yu F, Zhang Y, Zhao MH (2008) Antineutrophil cytoplasmic autoantibody-associated vasculitis in older patients. Medicine (Baltimore) 87 203-209. 